\title{
HYPONATREMIC SEIZURE IN AN AUTISTIC CHILD ASSOCIATED WITH EXCESSIVE WATER INTAKE WITH HERBAL MEDICINE
}

\author{
Manoj Kumar Jangid ${ }^{1}$, Nilesh Panchal ${ }^{1}$, Patlolla Nitin Reddy ${ }^{2}$. \\ ${ }^{1}$ Department of Pediatrics, NMC Royal Hospital, Dubai, United Arab Emirates, ${ }^{2}$ Department of Pediatrics, \\ London Neonatal Transfer Service, London, UK.
}

\begin{abstract}
Self-treatment with herbal remedies is a common practice, both in healthy individuals and those with a medical problem with a belief of safe alternatives to prescription medicines. The same is true in the case of autistic spectrum disorder (ASD) where many parents try different medicines in an attempt to improve overall health and behavioral problems. We present a 12 -year-old autistic boy who developed seizures due to dilutional hyponatremia following the use of an herbal mixture with excessive water. He was managed with fluid restriction and had a complete recovery. This case highlights the potential dyselectrolytemia associated with practice of excessive water intake with some of the herbal medication and the need for clinicians to be aware of such practice among families.
\end{abstract}

\section{Introduction}

Herbal and other plant-derived remedies have been estimated by the World Health Organization (WHO) to be the most frequently used therapies worldwide. ${ }^{1}$ Majority of the population using these herbal medicines are ignorant of their potential toxicities because of the erroneous beliefs that these medicines which are made directly from plants are natural and harmless. Excessive water intake along with these herbals, as a way of 'purifying and cleansing' the body is also a popular regime with the belief that harmful waste products can thus be washed from the body. ${ }^{2}$ We present a 12 years old autistic boy whose self-treatment by parents with an herbal mixture along with excessive water intake led to dilutional hyponatremia and seizures.

\section{Case Report}

A 12-year old autistic boy was rushed to emergency department (ED) by parents with an episode of generalized tonic clonic seizure 10 minutes prior at home which self-terminated. The boy had two episodes of vomiting the night before along with poor sleep and irritability. There was no history of fever, trauma, or similar episodes previously. The neurological and physical examination including vitals were unremarkable except brief period of postictal state. Child's initial routine investigations were within the normal range except low blood urea $9.0 \mathrm{mg} / \mathrm{dL}$ (Normal: $17-43 \mathrm{mg} / \mathrm{dL}$ ), low serum sodium $125 \mathrm{mEq} / \mathrm{L}$ (Normal: 136-146 mEq/L) and low chloride $90 \mathrm{mEq} / \mathrm{L}$ (Normal: 101-109 mEq/L). Post admission child was noticed to have high urine

CONTACT Dr. Manoj Kumar Jangid

Email: drmanojjangid@gmail.com

Address for Correspondence: Dr. Manoj Kumar Jangid, MD, Specialist Pediatrics, NMC Royal Hospital, Dubai Investments Park, Dubai, United Arab

Emirates.

(C)2020 Pediatric Oncall output ( $8 \mathrm{ml} / \mathrm{kg} / \mathrm{hr}$ ). On further inquiry, parents informed that they had increased his water intake from the past 5 days and were giving an herbal mixture with a belief of detoxifying the child's body in an attempt to reduce the autistic behavior. The contents of herbal medicine were not known but it was a mixture of 5-6 raw herbals as advised to the family on social forum for autistic children. They were also giving plain warm water enema 2-3 times per week and several dietary modifications in view of autism. Repeat investigations after 4 hours revealed serum sodium $132 \mathrm{mEq} / \mathrm{L}$, reduced serum osmolarity of $270 \mathrm{mOsm} / \mathrm{kg}$ (Normal: 285-295 mOsm/kg). Serum potassium and blood gas analysis were normal. Urine specific gravity was 1.002 . Urine osmolarity was $69 \mathrm{mOsm} / \mathrm{kg}$ (Normal: 300-900 $\mathrm{mOsm} / \mathrm{kg}$ ). Thus, a diagnosis of dilutional hyponatremia leading to seizure was considered and the child was kept on fluid restriction without any anticonvulsant. He improved over the next 18 hours, had no more vomiting, seizures and urine output returned to normal. The patient was discharged home the next day in a stable condition with a serum sodium level of $137 \mathrm{Eq} / \mathrm{L}$ and serum osmolarity of $286 \mathrm{mOsm} / \mathrm{kg}$. Over the next 12 months follow up, the child remained seizure-free and had no polydipsia.

\section{Discussion}

Herbal medicines are widely used self-medications. Complementary and alternative medicines are becoming increasingly popular, not only among the families of children with autism but also in several other pathological conditions. Some of the reasons for this increase interest in such therapies are nonavailability of effective therapies for ASD, parental perception of potential side effects with conventional drugs and considering these alternative treatments to be more safe and secure. Occasionally they may produce undesirable effects and some of them may be life-threatening. ${ }^{3}$ The potential toxicities result either due to the direct effect of the herb itself or due 
to adulteration of such complementary products with undeclared drugs. Adverse events also arise due to the medication changes made by alternative medicine practitioners or with some of the unusual practices associated with it like dietary restriction or excessive water intake. ${ }^{4}$ In our patient excessive water intake over a short period associated with intake of herbal mixture resulted in dilutional hyponatremia leading to seizure episode. . Unfortunately, the herbal mixture was used up and could not be recovered for toxicological investigations, so nothing could be concluded about the potential side effect related to the herb itself.

The usual clinical symptoms of hyponatremia ranges from headache, blurred vision, polyuria, vomiting, tremor to muscle cramps, ataxia, delirium, stupor, coma, and convulsions. ${ }^{5}$ Hyponatremia in autistic patients is often due to psychogenic polydipsia (PPD) and/or syndrome of inappropriate secretion of antidiuretic hormone (SIADH) from antidepressant medications or due to compulsive water drinking. ${ }^{6,7}$ Our patient was not on any antidepressant medications and there was no history suggestive of polydipsia. An extensive literature search revealed no previous reports of dilutional hyponatremia resulting from excessive water intake practiced with herbal therapy in children with autism. This case report highlights the risk of water intoxication leading to hyponatremia due to practice of excessive water intake with some herbal medicines. The patient's ongoing self-treatment with plain water enema abuse may have contributed to a lower seizure threshold. ${ }^{8}$ Although neurologic homeostasis can be adequately maintained in chronically hyponatremic patients, such children are prone to develop symptomatic hyponatremia after a relatively small increment in total body water. ${ }^{9}$

Many users of herbal medicines do not disclose about intake of herbals to their doctor as they consider them to natural and harmless. In the U.S., nearly $70 \%$ of patients who use herbal medicines do not inform their health care providers about their use of herbal therapies. ${ }^{10}$ So it is especially important to specifically ask in for the history of any medicinal product including traditional medicines and health supplements specially in chronic medical conditions-something that is often overlooked. Serum electrolyte concentrations should always be measured in children with afebrile seizures. Recognition of the condition will prevent inappropriate investigations and treatment.

\section{Conclusion}

We conclude that the practice of self-treatment with excessive water intake with herbal medicines with a belief of detoxifying the body represents a potential threat to the health of the children and may lead to water intoxication. An awareness of these facts is essential for the parents to understand the risk involved with such non evidence-based practices.

\section{Compliance with Ethical Standards \\ Funding: None \\ Conflict of Interest: None}

\section{References:}

1. Marini-Bettolo GB. Present aspects of the use of plants in traditional medicine. J Ethnopharmacol 1980;2:183-8.

2. Toovey OT, Edmond IR, Makris N. Acute severe hyponatremia secondary to polydipsia and associated herbal remedy use. BMJ Case Rep. 2016;2016:bcr2016216348.

3. Ekor M. The growing use of herbal medicines: issues relating to adverse reactions and challenges in monitoring safety. Front Pharmacol. 2014;4:177.

4. Lim A, Cranswick N, South M. Adverse events associated with the use of complementary and alternative medicine in children. Arch Dis Child. 2011;96(3):297-300.

5. Ferrier IN. Water intoxication in patients with psychiatric illness. Br Med J (Clin Res Ed). 1985;291:1594-6.

6. Chiang $\mathrm{CL}$, Lin $\mathrm{YH}$, Hsieh $\mathrm{MH}$. Olanzapine-induced hyponatremia in a patient with autism. J Child Adolesc Psychopharmacol. 2013;23:699-700

7. Hiratani M, Munesue T, Terai K, Haruki S. [Two cases of infantile autism with intermittent water intoxication due to compulsive water drinking and episodic release of antidiuretic hormone (SIADH)]. No To Hattatsu. 1997; 29: 367-372

8. Ziskind A, Gelis SS. Water intoxication following tap water enemas. J Dis Child. 1958;96:699-704

9. Arieff AI, Guisado R. Effects on the central nervous system of hypernatremic and hyponatremic states. Kidney Int. 1976;10:104-116

10. Eisenberg DM, Kessler RC, Foster C. Unconventional medicine in the United States: prevalence, costs, and patterns of use. N Engl J Med1993; 328:246-252. 